\title{
Expression of the Hippo transducer TAZ in association with WNT pathway mutations impacts survival outcomes in advanced gastric cancer patients treated with first-line chemotherapy
}

Elisa Melucci ${ }^{1 \dagger}$, Beatrice Casini ${ }^{1 \dagger}$, Livia Ronchetti ${ }^{1 \dagger}{ }^{1}$, Laura Pizzuti ${ }^{2}$, Francesca Sperati ${ }^{3}$, Matteo Pallocca ${ }^{4}$, Francesca De Nicola ${ }^{4}$, Frauke Goeman ${ }^{5}$, Enzo Gallo', Carla Azzurra Amoreo', Domenico Sergi², Irene Terrenato ${ }^{3}$, Patrizia Vici², Luigi Di Lauro², Maria Grazia Diodoro' ${ }^{2}$ Edoardo Pescarmona', Maddalena Barba²,6, Marco Mazzotta ${ }^{7}$, Marcella Mottolese ${ }^{1}$, Maurizio Fanciulli ${ }^{4}$, Gennaro Ciliberto ${ }^{6}$, Ruggero De Maria ${ }^{8 *}$, Simonetta Buglioni ${ }^{1 \neq}$ and Marcello Maugeri-Saccà $2,6^{*} \ddagger$

\begin{abstract}
Background: An extensive crosstalk co-regulates the Hippo and Wnt pathway. Preclinical studies revealed that the Hippo transducers YAP/TAZ mediate a number of oncogenic functions in gastric cancer (GC). Moreover, comprehensive characterization of GC demonstrated that the Wnt pathway is targeted by oncogenic mutations. On this ground, we hypothesized that YAP/TAZ- and Wnt-related biomarkers may predict clinical outcomes in GC patients treated with chemotherapy.

Methods: In the present study, we included 86 patients with advanced GC treated with first-line chemotherapy in prospective phase II trials or in routine clinical practice. Tissue samples were immunostained to evaluate the expression of YAP/TAZ. Mutational status of key Wnt pathway genes (CTNNB1, APC and FBXW7) was assessed by targeted DNA next-generation sequencing (NGS). Survival curves were estimated and compared by the Kaplan-Meier product-limit method and the log-rank test, respectively. Variables potentially affecting progression-free survival (PFS) were verified in univariate Cox proportional hazard models. The final multivariate Cox models were obtained with variables testing significant at the univariate analysis, and by adjusting for all plausible predictors of the outcome of interest (PFS).
\end{abstract}

\footnotetext{
*Correspondence: demariaruggero@gmail.com;

marcello.maugerisacca@ifo.gov.it

†Elisa Melucci, Beatrice Casini and Livia Ronchetti contributed equally to

this work

‡Simonetta Buglioni and Marcello Maugeri-Saccà share senior authorship

${ }^{6}$ Scientific Direction, "Regina Elena" National Cancer Institute, Via Elio Chianesi 53, 00144 Rome, Italy

${ }^{8}$ Institute of General Pathology, Catholic University of the Sacred Heart, Largo Agostino Gemelli, 10, 00168 Rome, Italy

Full list of author information is available at the end of the article
} 
Results: We observed a significant association between TAZ expression and Wnt mutations (Chi-squared $p=0.008$ ). Combined TAZ expression and Wnt mutations (TAZ ${ }^{\text {pos }} / \mathrm{WNT}^{\mathrm{mut}}$ ) was more frequently observed in patients with the shortest progression-free survival (negative outliers) (Fisher $p=0.021$ ). Uni-and multivariate Cox regression analyses revealed that patients whose tumors harbored the TAZ ${ }^{\text {pos }} / W N T^{\text {mut }}$ signature had an increased risk of disease progression (univariate Cox: HR 2.27, 95\% Cl 1.27-4.05, $p=0.006$; multivariate Cox: HR 2.73, 95\% Cl 1.41-5.29, $p=0.003$ ). Finally, the TAZ ${ }^{\text {pos }} / W N T^{\text {mut }}$ signature negatively impacted overall survival.

Conclusions: Collectively, our findings indicate that the oncogenic YAP/TAZ-Wnt crosstalk may be active in GC, conferring chemoresistant traits that translate into adverse survival outcomes.

Keywords: Gastric cancer, Hippo pathway, YAP, TAZ, Wnt pathway, CTNNB1, APC, FBXW7

\section{Background}

Over the past two decades, a wave of studies in flies elucidated the central role of the Hippo pathway in organ development [1]. Ablation of a set of genes including Warts (wts), Hippo (hpo), Salvador (sav) and Mob as tumor suppressor (mats) led to a remarkable tissue overgrowth, a process tied to increased cellular proliferation and reduced apoptosis [2-11]. These alterations were phenocopied upon the forced over-expression of the transcriptional co-activator Yorkie $(y k i)$ [12]. Complemented by functional and biochemical evidence, studies in Drosophila deciphered the functional architecture of the "Salvador-Warts-Hippo" (SWH) pathway, and have been instrumental for characterizing the Hippo pathway in mammals. Indeed, the use of conditional knockout alleles and inducible transgenic mice revealed that manipulation of Hippo pathway components resulted in tissue overgrowth and tumorigenesis [13, 14]. Functionally, Hippo is organized into a core regulatory module and a transcriptional module. The first is composed by the kinases sterile 20-like kinase 1 and 2 (MST1 and MST2; Hpo in Drosophila) and large tumor suppressor 1 and 2 (LATS1 and LATS2, Wts in Drosophila), together with the adaptor proteins Salvador homolog 1 (SAV1; Sav in Drosophila) and MOB kinase activator 1A and 1B (MOB1A and MOB1B; Mats in Drosophila). The latter encompasses the transcriptional cofactors yes-associated protein and its paralog transcriptional co-activator with PDZ-binding motif (YAP and TAZ, respectively; Yki in Drosophila), along with their transcriptional partners TEA domain-containing sequence-specific transcription factors (TEAD1-4; Scalloped in Drosophila) [1]. The core module orchestrates a phosphorylation cascade that results in the inhibition of YAP/TAZ, promoting their nuclear exclusion, cytoplasmic retention and proteasomal degradation [14-18]. When inactivated, or in the presence of stimuli that bypass its function, YAP/ TAZ accumulate into the nucleus, interact with their transcriptional partners and ultimately promote the transcription of target genes. Given that loss-of-function of Hippo kinases and adaptors fuelled tumor formation in animal models, and a similar outcome was observed upon the forced expression of Hippo transducers, Hippo was designated as a tumor suppressive signaling deputed to inhibit the oncogenic proteins YAP and TAZ [1].

Hippo signaling lies at the centerpiece of an intricate molecular network $[19,20]$. Indeed, a number of regulatory branches modulate its activity, spanning from cell polarity and cell adhesion factors to kinases acting upstream the regulatory module, mechanical forces (mechanotransduction), G-protein-coupled receptors (GPCRs) and metabolic routes [1]. An emerging level of regulation refers to the cooperation between Hippo and the Wnt pathway [1]. Central in the regulation of the Wnt signaling is the $\beta$-catenin destruction complex [21]. This is composed by a set of proteins that, in the absence of Wnt ligand stimulation, retains $\beta$-catenin in the cytoplasm and enables its degradation, thus preventing $\beta$-catenin nuclear translocation and transcription of target genes [21]. The crosstalk between Hippo and Wnt prevalently takes place at the level of $\beta$-catenin regulation [22, 23]. Two not mutually exclusive models have been proposed that functionally concatenate these two pathways. The first envisions the incorporation of YAP/ TAZ in the $\beta$-catenin destruction complex [22]. When the Wnt pathway is in the off state, YAP/TAZ participate in $\beta$-catenin degradation, whereas stimulation by Wnt ligands disassembles the complex promoting nuclear accumulation of both YAP/TAZ and $\beta$-catenin [22]. The second model proposes that Adenomatosis Polyposis Coli (APC), a central component of the $\beta$-catenin destruction complex, serves as a scaffold protein whose correct function is instrumental for the activation of Hippo kinases and consequent inhibition of YAP/TAZ [23]. Consistently, loss of APC disables Hippo-mediated control of YAP/TAZ [23].

Functional in vitro and in vivo studies linked aberrant activation of YAP/TAZ to the progression of gastric cancer (GC) [24], and the inhibition of the YAP/TAZ-TEAD interaction achieved with a Vgl-like-4-(VGLL4) mimicking peptide severely impaired GC cell survival [25]. Moreover, the comprehensive characterization of GC 
carried out by The Cancer Genome Atlas (TCGA) network revealed oncogenic mutations in central Wnt pathway components, including CTNNB1 ( $\beta$-catenin), APC and $F B X W 7$ (F-box/WD repeat domain-containing 7), an antagonist of the Wnt signaling that targets $\beta$-catenin for degradation [26]. On this ground, we hypothesized that the Hippo-Wnt pathway crosstalk may be active in GC, conferring more aggressive molecular traits that translate into adverse survival outcomes. To test this hypothesis, tissue samples from $86 \mathrm{GC}$ patients treated with first-line chemotherapy, either in prospective phase II trials or in routine clinical practice [27-30], were retrospectively evaluated by immunohistochemistry (IHC) for assessing the expression of YAP and TAZ. Immunohistochemical characterization was integrated with targeted DNA nextgeneration sequencing (NGS) analysis of CTNNB1, APC and $F B X W 7$.

\section{Methods}

\section{Patients and treatment}

In the present study, we included 86 patients with histologically confirmed, inoperable locally advanced or metastatic cancer of the stomach or gastroesophageal junction who received first-line chemotherapy (August 2001-June 2015). Median follow-up was 11 months (IQR 5.5-20.5 months). Eligibility was defined by the following criteria: (i) available data on clinical features, administered therapies and treatment outcomes, (ii) complete data on protein biomarkers (YAP and TAZ), and (iii) complete data on Wnt pathway component mutations. Chemotherapy regimens and schedules are detailed in Additional file 1. Tumor responses were evaluated by Response Evaluation Criteria in Solid Tumors (RECIST) criteria v.1.1. Progression-free survival (PFS) was calculated as the time between the first cycle of chemotherapy and radiological evidence of disease progression or death due to any cause. Overall survival (OS) was computed as the time from the first cycle of chemotherapy to death from any cause, and as the time from diagnosis to death due to any cause. Written informed consents were obtained by all the participants. The study was conducted in accordance with the Declaration of Helsinki and approved by the Ethics Committee of the "Regina Elena" National Cancer Institute of Rome. This study adheres to the REMARK guidelines [31].

\section{Immunohistochemical assessment of YAP and TAZ}

The immunohistochemical assessment of YAP and TAZ was performed in formalin-fixed paraffin-embedded (FFPE) tissues from biopsies or surgical samples, and was carried out with the following antibodies: anti-YAP monoclonal antibody (MoAb) (H-9, Santa Cruz) at the dilution of 1:200 and anti-TAZ MoAb (M2-616, BD
Pharmingen) at the dilution of 1:400. Immunoreactions were revealed by a streptavidin-biotin enhanced immunoperoxidase technique (Super Sensitive MultiLink, Leica, Milan, Italy) in an automated autostainer (Bond III, Leica). YAP/TAZ expression was reported both in terms of percentage of tumor-expressing cells and staining intensity $(0=$ absent, $1+=$ weak, $2+=$ moderate, and $3+=$ strong). For tumors with both nuclear and cytoplasmic expression, staining intensity and percentage of tumor-expressing cells were independently assessed in, and reported for, the two cellular compartments. Tumors were classified as negative (YAP ${ }^{\text {neg }}$, TAZ $^{\text {neg }}$ ) or positive $\left(\right.$ YAP $^{\text {pos }}$, TAZ $^{\text {pos }}$ ) on the basis of cellular localization and percentage of tumor-expressing cells. YAP/TAZ positivity was defined as a distinct nuclear immunoreactivity in $\geq 20 \%$ of neoplastic cells, a classification comparable to that of our previous studies [32-34]. Representative examples of immunohistochemical expression of YAP/ TAZ is provided in Additional file 2. Immunoreactivity was evaluated by two investigators blinded to treatment outcomes (EM and LR), and discordant cases were reviewed by a third observer (MM).

\section{Targeted DNA NGS}

DNA was extracted from FFPE tumor blocks using the QIAamp DNA Mini Kit (Qiagen). Quantity of the extracted DNA was assessed by the Qubit dsDNA High Sensitivity Assay Kit on Qubit Fluorometer (Thermofisher Scientific). Library preparation was performed on 20 ng DNA by the Ion AmpliSeq Library 96LV Kit 2.0 (Thermofisher Scientific) and the Wnt custom panel (Thermofisher Scientific), which targets 3 genes $(A P C$, CTNNB1 e FBXW7) and generates 174 amplicons (mean coverage: $98.8 \%$ ). Each library was barcoded with the Ion Xpress Barcode Adapters 1-16 Kit (Thermofisher Scientific) and diluted to a final concentration of $100 \mathrm{pM}$; barcoded libraries were pooled in equimolar amount and diluted to $35 \mathrm{pM}$ for downstream template preparation. Template preparation was performed by the Ion Chef system (Thermofisher Scientific), which integrates library amplification, ISP recovery-enrichment and Chip loading. Sequencing was performed on Ion S5 system (Thermofisher Scientific) with the Ion 520 chip. Raw data were analyzed using the Torrent Suite Software v.5.2 (Thermofisher Scientific). The coverage analysis was performed using the coverage analysis plug-in v5.2.1.2. All cases had a number of mapped reads $>100.000$ and/or the average base coverage $>500 \times$. Polymorphic variants were filtered out exploiting the Ion Reporter Suite (Thermofisher Scientific). Nucleotide variants with an allele frequency less than $3 \%$ were not considered. All variants were manually reviewed with Integrative Genomics Viewer (IGV V.2.1, Broad Institute, Cambridge, Massachusetts, USA), and 
with the support of publically available datasets reporting on their established or predicted oncogenicity (i.e. COSMIC, OncoKB and Mutation Assessor via cBioPortal). All molecular analyses were carried out in tissue samples collected before the administration of first-line chemotherapy for advanced disease.

\section{Statistical analysis}

Descriptive statistics were computed for all the variables of interest (clinical, pathological and molecular). The relationship between categorical variables was assessed with the Pearson's Chi squared test of independence (2-tailed) or the Fisher exact test, depending upon the size of the groups compared. The Kaplan-Meier product-limit method and the log-rank test were used for estimating and comparing survival curves. Variables potentially affecting PFS were tested in univariate Cox proportional hazard models (ECOG-PS: 0 vs $1-2$; stage: locally advanced vs metastatic; localization: stomach vs junction; number of metastatic sites: 1 vs 2-3; peritoneal metastasis: no vs yes; first-line taxane-containing chemotherapy: no vs yes; $\gamma$-H2AX/pATM: negative/single positive vs double positive) [35]. The final multivariate Cox models were obtained with variables testing significant at the univariate analysis, and by adjusting for all plausible predictors of the outcome of interest (PFS). The related estimates were reported as hazard ratio (HR) and 95\% confident interval (CI). The consistency of the TAZ ${ }^{\text {pos/ }}$ $\mathrm{WNT}^{\text {mut }}$ model was assessed through a re-sampling without replacement method (internal validation). More specifically, 100 hundred, less-powered datasets were generated by randomly removing 20\% from the original sample. For each simulation, the univariate Cox model was repeated and the replication rate was calculated. Level of significance was defined at $\mathrm{p}<0.05$. Statistical analyses were carried out using SPSS version 21.0 (SPSS Inc., Chicago, Illinois, USA).

\section{Results}

\section{Baseline characteristics of the study participants}

Baseline characteristics of the 86 patients included in the present study are summarized in Table 1. Median age at diagnosis was 61 years (IQ range 53.6-67.6). 36 (41.9\%) and $50(58.1 \%)$ patients had a locally advanced or metastatic disease, respectively. 50 (58.1\%) patients received three-drug chemotherapy, and taxane-containing regimens were administered to $47(54.7 \%)$ patients. In this series, $42(48.8 \%)$ patients were treated within the context of prospective phase II trials. We did not record any significant association between the investigational biomarkers (YAP, TAZ, Wnt mutations) and basal clinical and pathological characteristics detailed in Table 1 (data available upon request).
Table 1 Baseline characteristics of gastric cancer patients included in this study $(\mathrm{N}=86)$

\begin{tabular}{|c|c|}
\hline Characteristics & $N(\%)$ \\
\hline Age at diagnosis, median (min-max) [IQ range] & $61(28-79)[53.6-67.6]$ \\
\hline \multicolumn{2}{|l|}{ Gender } \\
\hline Male & $44(51.2)$ \\
\hline Female & $42(48.8)$ \\
\hline \multicolumn{2}{|l|}{ ECOG PS } \\
\hline 0 & $45(52.3)$ \\
\hline $1-2$ & $41(47.7)$ \\
\hline \multicolumn{2}{|l|}{ Stage } \\
\hline Locally advanced & $36(41.9)$ \\
\hline Metastatic & $50(58.1)$ \\
\hline \multicolumn{2}{|l|}{ Previous surgery } \\
\hline No & $33(38.4)$ \\
\hline Yes & $53(61.6)$ \\
\hline \multicolumn{2}{|l|}{ Neoadjuvant/adjuvant chemotherapy } \\
\hline No & $61(70.9)$ \\
\hline Yes & $25(29.1)$ \\
\hline \multicolumn{2}{|l|}{ Lauren classification } \\
\hline Intestinal & $35(40.7)$ \\
\hline Diffuse & $41(47.7)$ \\
\hline Mixed & $10(11.6)$ \\
\hline \multicolumn{2}{|l|}{ Grade } \\
\hline $\mathrm{G} 2$ & $21(24.4)$ \\
\hline G3 & $65(75.6)$ \\
\hline \multicolumn{2}{|l|}{ Localization } \\
\hline Esophagogastric junction & $6(7.0)$ \\
\hline Stomach & $80(93.0)$ \\
\hline \multicolumn{2}{|l|}{ Agents (N) } \\
\hline 2 & $36(41.9)$ \\
\hline 3 & $50(58.1)$ \\
\hline \multicolumn{2}{|l|}{ Taxanes (first-line) } \\
\hline No & $39(45.3)$ \\
\hline Yes & $47(54.7)$ \\
\hline
\end{tabular}

\section{Relationship between YAP/TAZ expression and Wnt mutations}

The individual distribution of the molecular biomarkers evaluated in the present study is illustrated in Fig. 1a. YAP and TAZ positivity was observed in 76 (88.4\%) and $35(40.7 \%)$ tumor samples, respectively. Mutations of CTNNB1, APC and FBXW7 were detected in 9 (10.5\%), 20 (23.2\%), and $17(19.8 \%)$ tumor samples, respectively. When considering integrated pathway analysis, 30 (34.9\%) tumors carried at least one mutations in Wnt pathway genes (Fig. 1a). Overall, 95 mutations were detected: CTNNB1 $\mathrm{N}=14, A P C \mathrm{~N}=45$ and $F B X W 7 \mathrm{~N}=36$ (Fig. 1b). In the search of predictive factors, we first sought to address the relationship between 


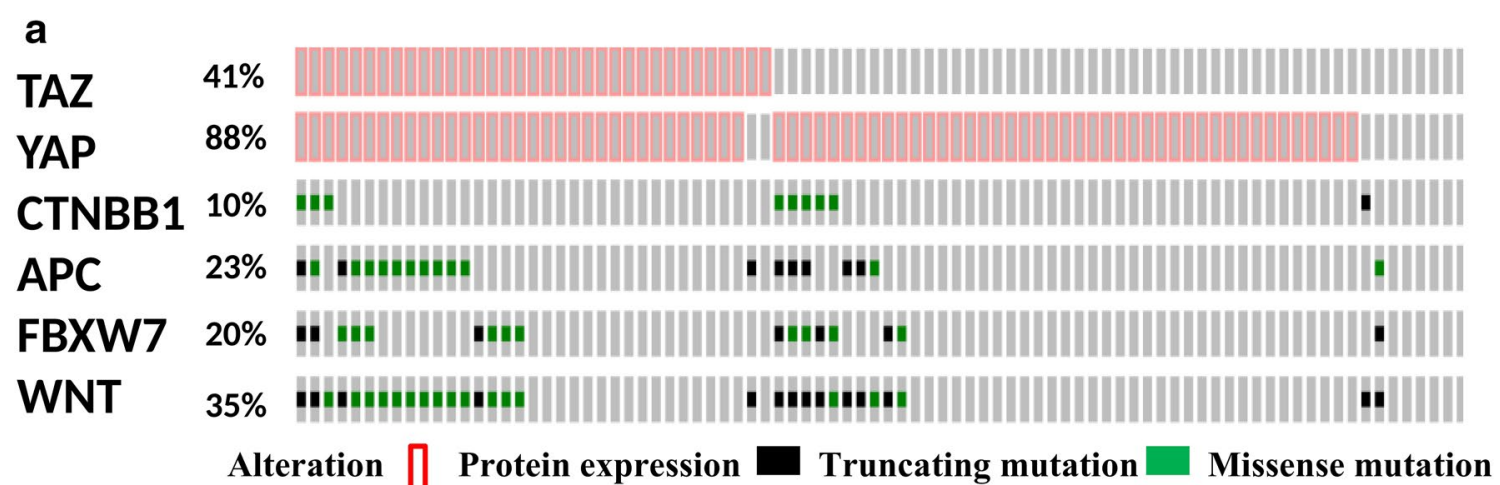

b

APC

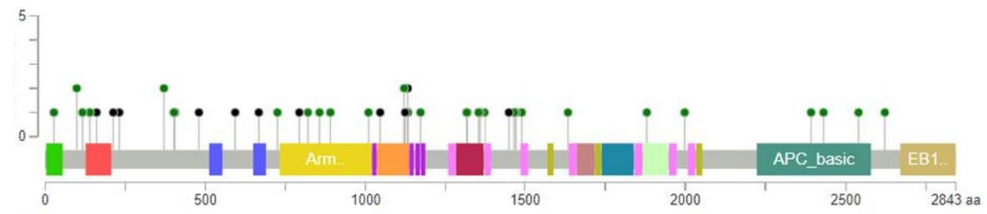

31 Missense $\quad 14$ Truncating

CTNNB1

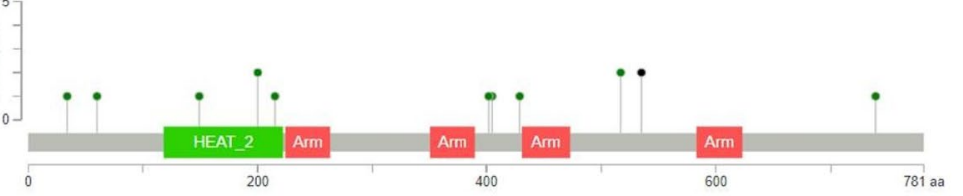

13 Missense $\quad 1$ Truncating

\section{FBXW7}

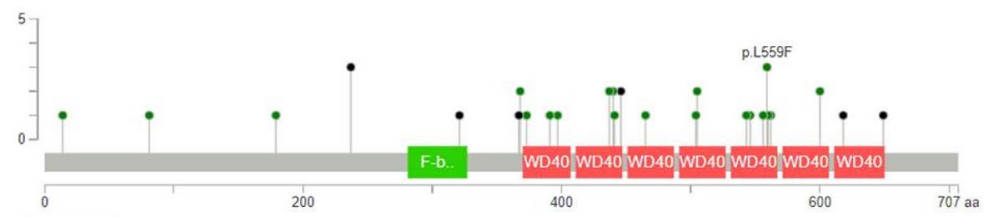

27 Missense $\quad 9 \quad$ Truncating

Fig. 1 a Oncoprint showing the distribution of the investigated biomarkers (YAP, TAZ, CTNNB1, APC, FBXW7) together with the cases with at least one mutations in Wnt pathway components (integrated pathway analysis). b MutationMapper illustrating the entire set of detected mutations (and their nature) represented on the linear proteins

the various investigational biomarkers. We observed a significant association between nuclear TAZ expression and the presence of Wnt mutations (Chi-squared $\mathrm{p}=0.008$ ) (Table 2). Prompted by this observation, we conducted an outlier analysis in the attempt of identifying molecular features that characterize exceptional responders and patients with intrinsically chemoresistant disease. Thus, we verified the distribution of YAP, TAZ, and Wnt mutations in negative and positive outliers, defined as patients in the lowest (PFS $<3.3$ months) and highest (PFS $\geq 11.0$ months) quartile $(\mathrm{N}=43)$. When
Table 2 Association between the expression of YAP/TAZ and Wnt pathway mutations $(\mathrm{N}=86)$

\begin{tabular}{lrrl}
\hline & \multicolumn{1}{l}{ WNT } & & p-value \\
\cline { 2 - 3 } & WT & MUT & \\
\hline N (\%) & $\mathbf{N}(\%)$ & \\
\hline TAZ $^{\text {neg }}$ & $39(76.5)$ & $12(23.5)$ & 0.008 (Chi-squared) \\
YAP $^{\text {nos }}$ & $17(48.6)$ & $18(51.4)$ & \\
YAPpos $^{\text {pos }}$ & $7(70.0)$ & $3(30.0)$ & 0.999 (Fisher) \\
\hline
\end{tabular}


biomarkers were individually considered, only TAZ was significantly more expressed in the negative outlier group (Chi-squared $\mathrm{p}=0.044$ ) (Table 3). However, the strongest association was observed when we tested a signature that combined nuclear TAZ expression and Wnt mutations (TAZ ${ }^{\mathrm{pos}} / \mathrm{WNT}^{\mathrm{mut}}$ ), which was significantly overrepresented in the negative outlier group (Fisher $\mathrm{p}=0.021$ ) (Table 3). Considering the significant association between nuclear TAZ expression and Wnt pathway mutations (Table 2), results from the outlier analysis that indicate a different distribution of the $\mathrm{TAZ}^{\text {pos }} / \mathrm{WNT}^{\text {mut }}$ signature between negative and positive outliers (Table 3 ), and taking into account the biological plausibility of the TAZ ${ }^{\text {pos } / ~}$ $\mathrm{WNT}^{\text {mut }}$ model, this molecular profile was further investigated for its impact on survival outcomes.

\section{Association between the $\mathrm{TAZ}^{\text {pos }} / \mathrm{WNT}^{\text {mut }}$ signature and survival outcomes}

Patients whose tumors carried the TAZ ${ }^{\text {pos }} / \mathrm{WNT}^{\text {mut }}$ signature experienced significant shorter PFS compared with their negative counterparts $(\log$ rank $\mathrm{p}=0.004)$ (Fig. 2). In the univariate Cox regression analyses, the $\mathrm{TAZ}^{\mathrm{pos}} / \mathrm{WNT}^{\text {mut }}$ signature was the only variable associated with an increased risk of progression (HR 2.27, 95\% CI 1.27-4.05, $\mathrm{p}=0.006$ ) (Table 4 ), together with a DNA damage repair signature we previously developed in a larger cohort of $110 \mathrm{GC}$ patients (the $\gamma-\mathrm{H} 2 \mathrm{AX}^{\mathrm{pos}} / \mathrm{pATM}$ pos model) [35]. In the multivariate Cox models obtained by adjusting for all the plausible predictors tested in univariate analysis, the $\mathrm{TAZ}^{\mathrm{pos}} / \mathrm{WNT}^{\text {mut }}$ signature remained associated with an increased risk of disease progression (HR 2.73, 95\% CI 1.41-5.29, $\mathrm{p}=0.003$ ) (Table 4). Comparable results emerged when exclusively adjusting for the $\gamma-\mathrm{H}_{2} \mathrm{AX}^{\mathrm{pos}} / \mathrm{pATM}^{\mathrm{pos}}$ signature $(\mathrm{HR} 2.21,95 \% \mathrm{CI}$

Table 3 Association between Hippo- and Wnt-related biomarkers and positive/negative outliers $(\mathrm{N}=43)$

\begin{tabular}{|c|c|c|c|}
\hline & \multicolumn{2}{|l|}{ PFS (outliers) } & \multirow{3}{*}{$\begin{array}{l}\text { Chi-squared test } \\
\text { p-value }\end{array}$} \\
\hline & $<1$ quartile & $>3$ quartile & \\
\hline & N (\%) & N (\%) & \\
\hline TAZ $Z^{\text {neg }}$ & $10(37.0)$ & $17(63.0)$ & 0.044 \\
\hline TAZ & $11(68.8)$ & $5(31.3)$ & \\
\hline YAPneg & $2(40.0)$ & $3(60.0)$ & $0.999^{*}$ \\
\hline YAPpos & $19(50.0)$ & $19(50.0)$ & \\
\hline WNTwt & $11(39.3)$ & $17(60.7)$ & 0.087 \\
\hline WNT ${ }^{\text {mut }}$ & $10(66.7)$ & $5(33.3)$ & \\
\hline Other & $14(40.0)$ & $21(60.0)$ & $0.021^{*}$ \\
\hline $\mathrm{TAZ}^{\mathrm{pos}} / \mathrm{WNT} \mathrm{T}^{\mathrm{mut}}$ & $7(87.5)$ & $1(12.5)$ & \\
\hline Other & $12(41.4)$ & $17(58.6)$ & 0.159 \\
\hline YAPpos$/ W N T^{\text {mut }}$ & $9(64.3)$ & $5(35.7)$ & \\
\hline
\end{tabular}

* Fisher's exact test
1.23-3.97, $\mathrm{p}=0.008$ ) (Table 4). Collectively, these data indicate that the $\mathrm{TAZ}^{\mathrm{pos}} / \mathrm{WNT}^{\text {mut }}$ signature confers an increased risk of disease progression, and suggest that two independent molecular predictors were identified $\left(\mathrm{TAZ}^{\mathrm{pos}} / \mathrm{WNT}^{\mathrm{mut}}\right.$ and $\gamma$-H2AX $\left.{ }^{\mathrm{pos}} / \mathrm{pATM}^{\mathrm{pos}}\right)$. Upon resampling (procedure detailed in "Statistical analyses" section), the replication rate for the univariate Cox model for PFS was $84 \%$, thus indicating the stability of the model. Finally, the TAZ ${ }^{\text {pos }} / \mathrm{WNT}^{\text {mut }}$ signature was associated with inferior overall survival, albeit to a not fully significant extent ( $\log$ rank $\mathrm{p}=0.076)$ (Fig. 3a). Nevertheless, this association became fully significant when OS was computed from the time of diagnosis instead of date at the initiation of chemotherapy $(\log$ rank $p=0.035)$ (Fig. 3b), suggesting that the TAZ ${ }^{\text {pos }} / \mathrm{WNT}^{\text {mut }}$ signature may hold both predictive and prognostic significance.

\section{Discussion}

In the present study, we examined the expression of the Hippo transducers YAP/TAZ together with mutations in central components of the Wnt pathway in a relatively large series of advanced GC patients treated with chemotherapy in the first-line setting. Approximately half of the patients examined were treated in the context of prospective phase II trials [27-30]. This study, which is hypothesis-generating by nature, capitalizes on a growing body of evidence that converge on assigning to the Hippo-Wnt pathway cooperation a central role in three intertwined processes, namely organ development, tissue repair after injuries and tumorigenesis [22, 23]. Collectively, our results indicate that: (i) a subset of GC is characterized by a signature denoting deregulation of both Hippo and Wnt, (ii) the coexistence of nuclear TAZ expression and pathogenic Wnt pathway mutations seems to be predictive of shorter PFS, and then reduced efficacy of first-line chemotherapy, and (iii) the TAZ ${ }^{\text {pos/ }}$ $\mathrm{WNT}^{\text {mut }}$ signature may also represent an adverse prognostic factor. To our knowledge, this is the first report striving to address the clinical significance of the HippoWnt crosstalk in GC. Earlier studies suggested that YAP/ TAZ are often expressed in GC, which is consistent with our data [24, 36-38]. Nevertheless, studies reported so far have described small-sized case series without a clear focus on therapeutic outcomes (e.g. by pooling data concerning patients with various disease stages and prognosis), or have been conducted in specific disease entities which are not necessarily representative for the overall category of advanced GC (e.g. signet ring cell carcinoma, gastroesophageal junction cancers) [24, 36-38].

In our opinion, our findings raised a number of points that may streamline the identification of Hippo/Wntrelated predictive factors in GC. First, the molecular characterization of GC delineated four distinct molecular 


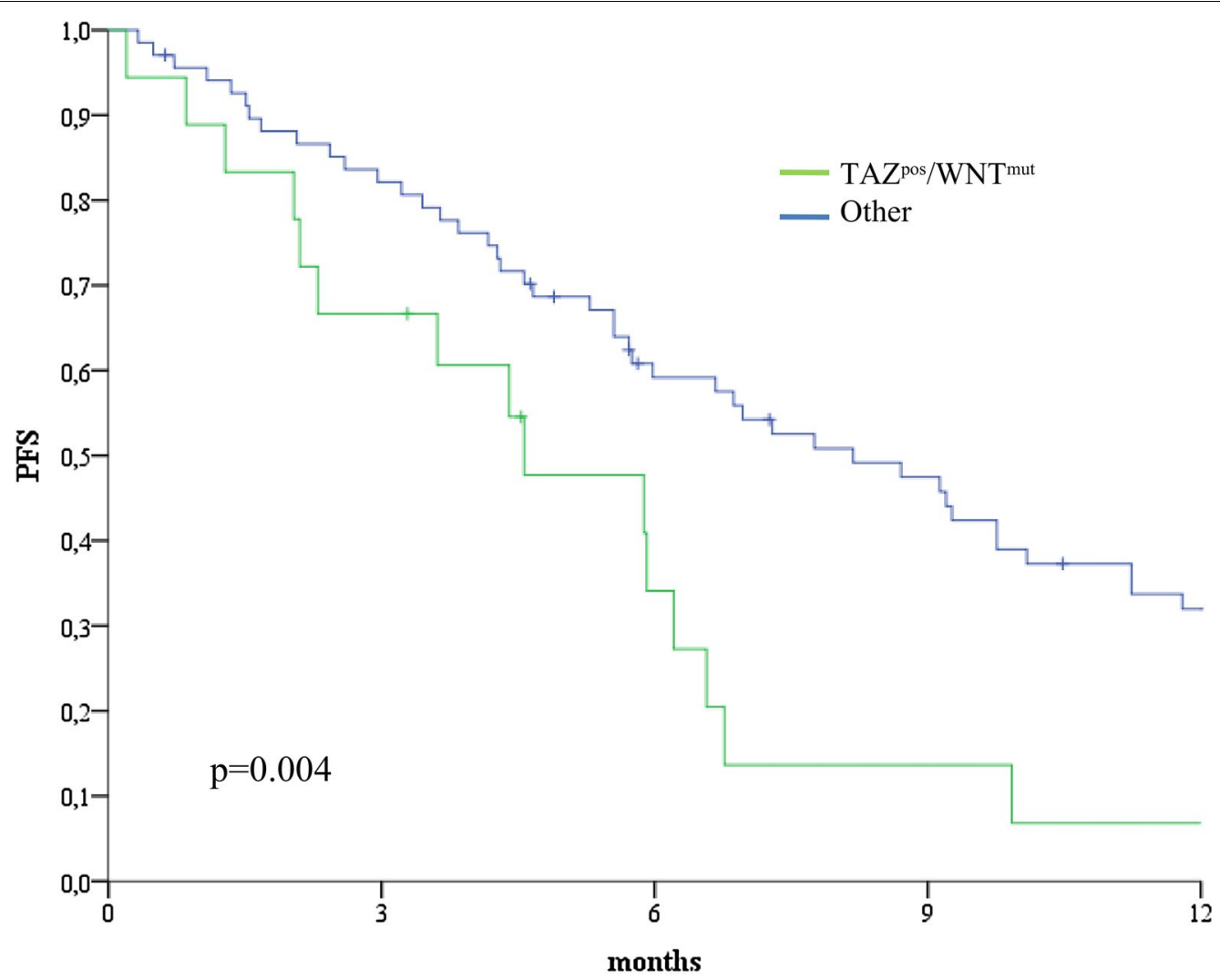

$\mathrm{N}$. of patients still at risk:

$\begin{array}{lrrrr}\text { Neg } & 55 & 36 & 28 & 18 \\ \text { Pos } & 12 & 5 & 2 & 1\end{array}$

Fig. 2 Kaplan-Meier survival curves of progression-free survival comparing TAZ ${ }^{\text {pos }} / \mathrm{WN}^{\mathrm{mut}}$ cases versus their negative counterparts $(\mathrm{N}=86)$

subtypes: chromosomal instability (CIN), microsatellite instability (MSI), genomically stable (GS) and EpsteinBarr virus (EBV)-positive [26]. Mutations in Wnt pathway components were observed across all non-hypermutated subtypes. Conversely, hallmarks of GS-GC are RHOA and $C D H 1$ mutations, together with CLDN18-ARHGAP26 fusions. All these alterations suggest geneticallydriven deregulation of the Hippo pathway. Indeed, Rho GTPases are involved in the activation of YAP/TAZ and in the inhibition of Hippo kinases via two distinct mechanisms: (i) stimulation by soluble factors that act through G-protein-coupled receptors (GPCRs) and Rho GTPases [39-43], and (ii) mechanical cues, such as extracellular matrix stiffness and changes in cell geometry, attachment status and density, that regulate YAP/TAZ through Rho GTPases and the remodeling of the F-actin cytoskeleton [44-46]. Next, CDH1 encodes for the cell-cell adhesion molecule E-cadherin, the central component of adherens junctions. E-cadherin is an established positive regulator of MST1/2 activity, whereas the E-cadherin-associated protein $\alpha$-catenin sequesters YAP/TAZ in the cytoplasm, hindering their nuclear translocation [47-49]. Consistently, disruption of the E-cadherin-catenin complex at the cell-cell junction fuels YAP/TAZ activation [47-49]. Finally, the CLDN18-ARHGAP26 fusion implies defects in CLDN18 and ARHGAP26. CLDN18 encodes for Claudin 18, a component of tight junctions (TJs) [50]. TJ proteins promote activation of Hippo kinases and/ or sequester YAP/TAZ in the cytoplasm, whereas $A R H$ GAP26 encodes for the Rho-Type GTPase-Activating Protein 26 [51-57]. These observations suggest that GS-GC is characterized by multiple defects in cell-cell adhesion mechanisms that, in turn, can propel YAP/TAZ activation. Different considerations apply to EBV-related GC. Experimental models of liver and cervical tumors are beginning to shed light on the connection between viral proteins and YAP/TAZ. For instance, the hepatitis B virus $\mathrm{X}$ protein $(\mathrm{HBx})$ up-regulates YAP promoting the growth 
Table 4 Uni- and multivariate Cox regression models for PFS ( $N=86$ )

\begin{tabular}{|c|c|c|c|c|c|c|}
\hline & \multicolumn{2}{|c|}{ Univariate Cox regression model } & \multicolumn{2}{|c|}{ Multivariate Cox regression model ${ }^{a}$} & \multicolumn{2}{|c|}{$\begin{array}{l}\text { Multivariate Cox regression } \\
\text { model }^{\mathbf{b}}\end{array}$} \\
\hline & $\mathrm{HR}(95 \% \mathrm{Cl})$ & p-value & HR $(95 \% \mathrm{Cl})$ & p-value & $\mathrm{HR}(95 \% \mathrm{Cl})$ & p-value \\
\hline \multicolumn{7}{|l|}{$\gamma-\mathrm{H}_{2} \mathrm{AX}^{\text {pos }} / \mathrm{pATM} \mathrm{M}^{\text {pos }}$} \\
\hline Positive vs other & $2.14(1.30-3.53)$ & 0.003 & $2.09(1.27-3.45)$ & 0.004 & $1.87(1.04-3.39)$ & 0.038 \\
\hline \multicolumn{7}{|l|}{ TAZ ${ }^{\text {pos }} / W N T^{\text {mut }}$} \\
\hline Positive vs other & $2.27(1.27-4.05)$ & 0.006 & $2.21(1.23-3.97)$ & 0.008 & $2.73(1.41-5.29)$ & 0.003 \\
\hline \multicolumn{7}{|l|}{ ECOG-PS } \\
\hline $1-2$ vs 0 & $1.23(0.77-1.97)$ & 0.391 & & & $1.20(0.73-1.95)$ & 0.471 \\
\hline \multicolumn{7}{|l|}{ Stage } \\
\hline Met vs loc adv & $1.12(0.69-1.80)$ & 0.647 & & & $0.89(0.46-1.73)$ & 0.737 \\
\hline \multicolumn{7}{|l|}{ Localization } \\
\hline Stomach vs EOJ & $0.67(0.27-1.69)$ & 0.398 & & & $1.54(0.53-4.42)$ & 0.424 \\
\hline \multicolumn{7}{|c|}{ Number of metastatic sites } \\
\hline $2-3$ vs 1 & $1.57(0.93-2.65)$ & 0.089 & & & $1.33(0.70-2.50)$ & 0.379 \\
\hline \multicolumn{7}{|c|}{ Peritoneal metastasis } \\
\hline Yes vs No & $0.67(0.42-1.07)$ & 0.097 & & & $0.72(0.41-1.27)$ & 0.261 \\
\hline \multicolumn{7}{|l|}{ Taxanes } \\
\hline Yes vs No & $0.93(0.58-1.51)$ & 0.784 & & & $0.79(0.45-1.40)$ & 0.429 \\
\hline
\end{tabular}

a Adjusted for the variables significant at the univariate analysis

b Adjusted for all the variables tested at univariate analysis

of hepatoma cells, whereas in hepatocellular carcinoma cell lines the transcriptional activator PreS2 up-regulates TAZ via the suppression of miRNA-338-3p $[58,59]$. Likewise, in cervical cancer cells the HPV E6 protein protects YAP from proteasome-dependent degradation in a process that ignites cancer cell proliferation [60]. Remarkably, a distinctive feature of EBV-associated GC is the extreme DNA hypermethylation, and both $M O B 1 B$ and WWTR1 (the gene encoding for TAZ) present frequent promoter hypermethylation [26]. Thus, more tailored investigations are needed in the future, which specifically take into account the molecular classification of GC and the underlying molecular portraits characterizing the different subtypes.

Another aspect that deserves mention is that the activity of Hippo and Wnt is modulated by negative feedback loops. Indeed, the YAP/TAZ-TEADs and $\beta$-cateninTCF/LEF complexes also promote the transcription of negative pathway regulators [61, 62]. For instance, the YAP/TAZ-TEADs complex controls the activity of Hippo kinases by inducing the expression of LATS2, and mediates the transcription of neurofibromin 2 (NF2, also known as Merlin), an established positive regulator of LATS1/2 kinase activity [61]. Aware of these mechanisms, our original experimental workflow envisioned targeted RNA sequencing for evaluating two signatures denoting the activation of YAP/TAZ and Wnt. The logic behind this was to carry out an extensive characterization at three different levels (protein, transcript and gene), which would have enabled us to investigate negative feedback loops. Even though this task was halted owing to excessive RNA degradation in the majority of samples, transcript-level analysis will be further pursued in future studies from our research team.

Finally, Hippo and Wnt are two pieces of a wider crossregulation process involving multiple signaling pathways [i.e. Hedgehog, Notch and Bone Morphogenetic Protein (BMP)], whose activity is central in organ development, tissues homeostasis, stem cell fate and tumorigenesis [19, 20]. Albeit these pathways are not targeted by genetic events in GC [26], the evaluation of biomarkers functioning as readout for their activation may add further granularity, allowing the evaluation of co-regulated signaling avenues.

\section{Conclusions}

Our data pointed to the combined activation of two oncogenic avenues, YAP/TAZ and Wnt, as potential biomarkers for predicting the efficacy of chemotherapy in GC patients. Considering the intricate molecular network 


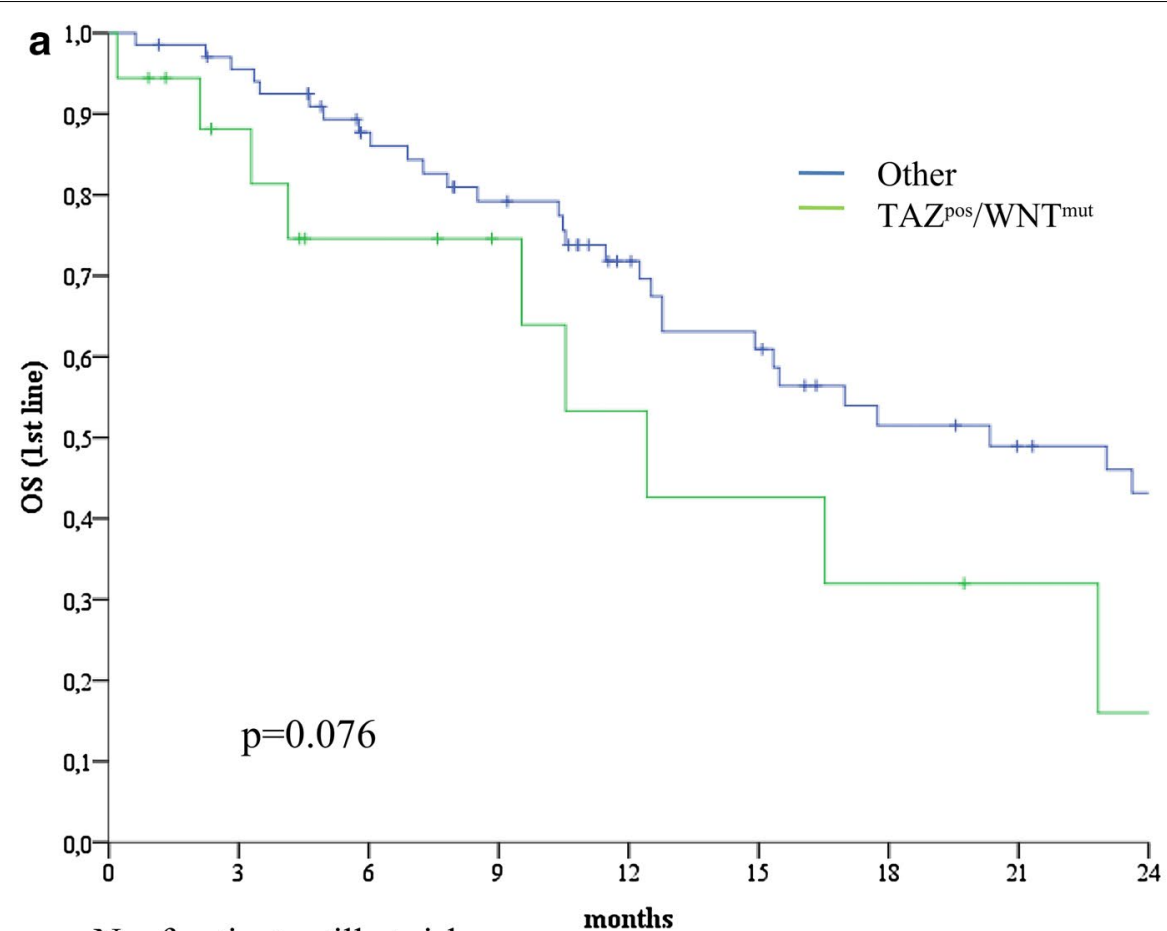

N. of patients still at risk:

$\begin{array}{lrrrrrrrr}\text { Neg } & 63 & 52 & 45 & 34 & 28 & 21 & 18 & 15 \\ \text { Pos } & 13 & 9 & 7 & 5 & 4 & 3 & 2 & 1\end{array}$

b

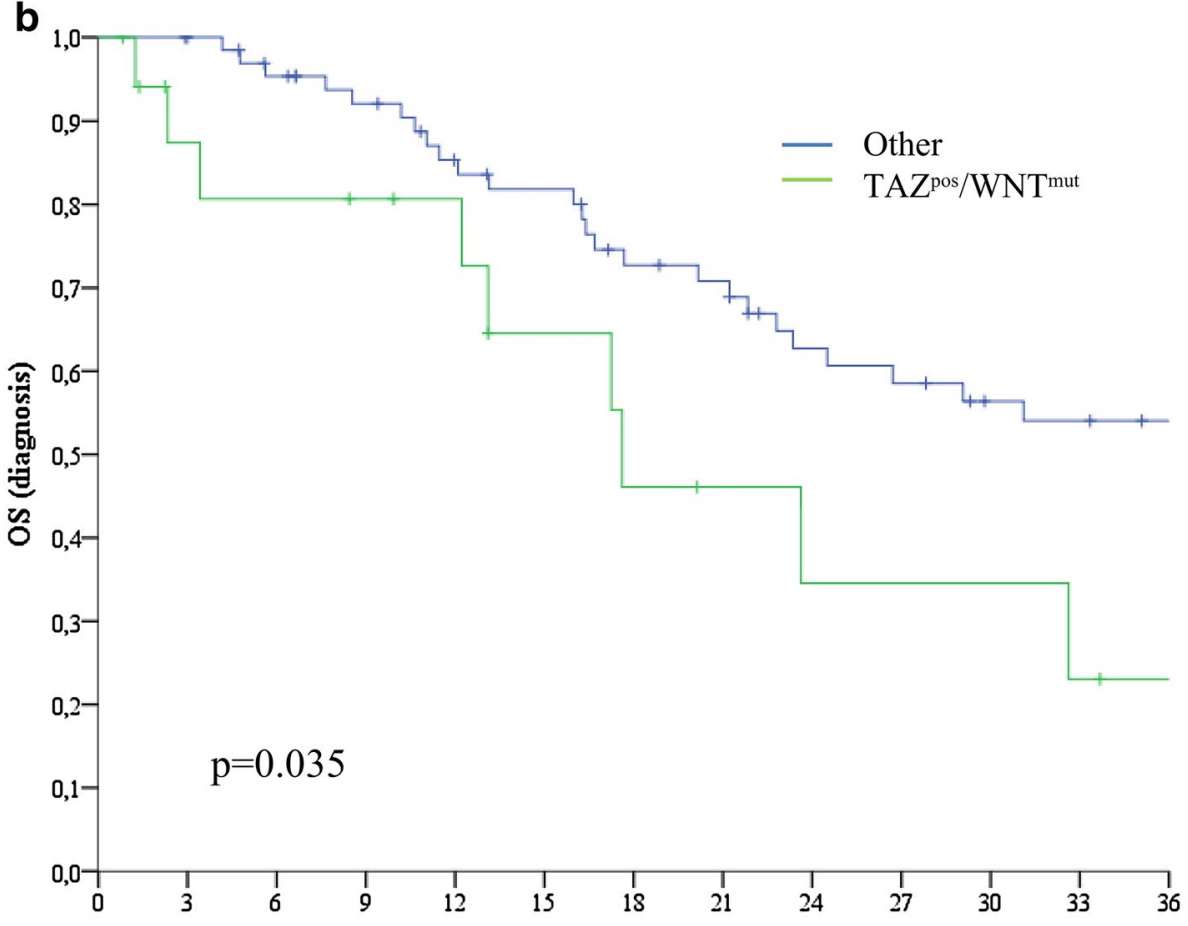

N. of patients still at risk:

months

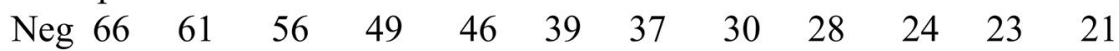

$\begin{array}{lllllllllllll}\text { Pos } & 13 & 12 & 11 & 10 & 7 & 5 & 4 & 3 & 3 & 3 & 2 & 1\end{array}$

Fig. 3 Kaplan-Meier survival curves of overall survival comparing TAZ ${ }^{\text {pos }} / W N T^{\text {mut }}$ cases versus their negative counterparts ( $\left.N=86\right)$. a Refers to overall survival calculated from the first cycle of chemotherapy, whereas $\mathbf{b}$ illustrates overall survival calculated from diagnosis 
that co-regulates YAP/TAZ and Wnt, we believe that more comprehensive, subtype-restricted, pathway analyses will be instrumental to gain a better understanding on the Hippo-Wnt pathway crosstalk and its clinical implications.

\section{Additional files}

Additional file 1. First-line chemotherapy regimens and schedules $(\mathrm{N}=86)$.

Additional file 2. Representative examples of immunohistochemical expression of TAZ and YAP in gastric cancer. Two cases are presented with combined nuclear expression of both TAZ and YAP (A-D).

\section{Abbreviations}

APC: Adenomatosis Polyposis Coli; CTNNB1: $\beta$-catenin; FBXW7: F-box/WD repeat domain-containing 7; GC: gastric cancer; GPCRs: G-protein-coupled receptors; LATS1 and LATS2: large tumor suppressor 1 and 2; MOB1A and MOB1B: MOB kinase activator 1A and 1B; MST1 and MST2: sterile 20-like kinase 1 and 2; OS: overall survival; PFS: progression-free survival; SAV1: Salvador homolog 1; TAZ: transcriptional co-activator with PDZ-binding motif; TEAD1-4: TEA domain-containing sequence-specific transcription factors; YAP: yesassociated protein.

\section{Authors' contributions}

$\mathrm{SB}, \mathrm{GC}, \mathrm{RDM}$ and $\mathrm{MM}-\mathrm{S}$ conceived and designed the study. EM, BC, LR, FDN, $F G, E G, C A A, M M, M F$ and $S B$ carried out molecular pathology analyses. $L P$, $D S, P V, L D L, M G D, E P$ and $M M$ acquired and reviewed clinical and pathological data. FS, MP, IT and MB performed bioinformatic and statistical analyses. All authors have been involved in drafting the manuscript. MM-S wrote the manuscript. All authors agree to be accountable for all aspects of the work. All authors read and approved the final manuscript

\section{Author details}

${ }^{1}$ Department of Pathology, "Regina Elena” National Cancer Institute, Via Elio Chianesi 53, 00144 Rome, Italy. ${ }^{2}$ Division of Medical Oncology 2, "Regina Elena" National Cancer Institute, Via Elio Chianesi 53, 00144 Rome, Italy. ${ }^{3}$ Biostatistics-Scientific Direction, "Regina Elena" National Cancer Institute, Via Elio Chianesi 53, 00144 Rome, Italy. ${ }^{4}$ SAFU Laboratory, Department of Research, Advanced Diagnostic, and Technological Innovation, "Regina Elena"National Cancer Institute, Via Elio Chianesi 53, 00144 Rome, Italy. ${ }^{5}$ Oncogenomic and Epigenetic Unit, "Regina Elena" National Cancer Institute, Via Elio Chianesi 53, 00144 Rome, Italy. ${ }^{6}$ Scientific Direction, "Regina Elena" National Cancer Institute, Via Elio Chianesi 53, 00144 Rome, Italy. ${ }^{7}$ Medical Oncology Unit, Policlinico Sant'Andrea, Via Di Grotta Rossa, 1035/1039, 00189 Rome, Italy. ${ }^{8}$ Institute of General Pathology, Catholic University of the Sacred Heart, Largo Agostino Gemelli, 10, 00168 Rome, Italy.

\section{Acknowledgements}

We thank Tania Merlino for editorial assistance. We also thank Salvatore Conti (Thermo Fisher Scientific) for technical support.

\section{Competing interests}

The authors declare that they have no competing interests.

\section{Availability of data and materials}

The datasets analyzed during the current study is available from the corresponding author on reasonable request.

\section{Consent for publication}

Not applicable.

\section{Ethics approval and consent to participate}

This study was approved by the Ethic Committee of "Regina Elena"National Cancer Institute. Written informed consents were obtained by all the participants. The study was conducted in accordance with the Declaration of Helsinki.

\section{Funding}

This study was supported by an intramural research grant to the "Gastrointestinal Tumors Translational Research Group".

\section{Publisher's Note}

Springer Nature remains neutral with regard to jurisdictional claims in published maps and institutional affiliations.

Received: 27 November 2017 Accepted: 11 January 2018

Published online: 05 February 2018

\section{References}

1. Piccolo S, Dupont S, Cordenonsi M. The biology of YAP/TAZ: hippo signaling and beyond. Physiol Rev. 2014;94:1287-312.

2. Justice RW, Zilian O, Woods DF, Noll M, Bryant PJ. The Drosophila tumor suppressor gene warts encodes a homolog of human myotonic dystrophy kinase and is required for the control of cell shape and proliferation. Genes Dev. 1995;9:534-46.

3. Xu T, Wang W, Zhang S, Stewart RA, Yu W. Identifying tumor suppressors in genetic mosaics: the Drosophila lats gene encodes a putative protein kinase. Development. 1995;121:1053-63.

4. Harvey KF, Pfleger CM, Hariharan IK. The Drosophila Mst ortholog, hippo, restricts growth and cell proliferation and promotes apoptosis. Cell. 2003;114:457-67.

5. Jia J, Zhang W, Wang B, Trinko R, Jiang J. The Drosophila Ste20 family kinase dMST functions as a tumor suppressor by restricting cell proliferation and promoting apoptosis. Genes Dev. 2003;17:2514-9.

6. Pantalacci S, Tapon N, Leopold P. The Salvador partner Hippo promotes apoptosis and cell-cycle exit in Drosophila. Nat Cell Biol. 2003:5:921-7.

7. Udan RS, Kango-Singh M, Nolo R, Tao C, Halder G. Hippo promotes proliferation arrest and apoptosis in the Salvador/Warts pathway. Nat Cell Biol. 2003;5:914-20.

8. Wu S, Huang J, Dong J, Pan D. Hippo encodes a Ste-20 family protein kinase that restricts cell proliferation and promotes apoptosis in conjunction with salvador and warts. Cell. 2003;1 14:445-56.

9. Kango-Singh M, Nolo R, Tao C, Verstreken $\mathrm{P}$, Hiesinger PR, Bellen $\mathrm{HJ}$, et al. Shar-pei mediates cell proliferation arrest during imaginal disc growth in Drosophila. Development. 2002;129:5719-30.

10. Tapon N, Harvey KF, Bell DW, Wahrer DC, Schiripo TA, Haber D, et al. Salvador promotes both cell cycle exit and apoptosis in Drosophila and is mutated in human cancer cell lines. Cell. 2002;1 10:467-78.

11. Lai ZC, Wei X, Shimizu T, Ramos E, Rohrbaugh M, Nikolaidis N, et al. Control of cell proliferation and apoptosis by mob as tumor suppressor, mats. Cell. 2005;120:675-85.

12. Huang J, Wu S, Barrera J, Matthews K, Pan D. The Hippo signaling pathway coordinately regulates cell proliferation and apoptosis by inactivating Yorkie, the Drosophila homolog of YAP. Cell. 2005;122:421-34.

13. Camargo FD, Gokhale S, Johnnidis JB, Fu D, Bell GW, Jaenisch R, et al. YAP1 increases organ size and expands undifferentiated progenitor cells. Curr Biol. 2007:17:2054-60.

14. Dong J, Feldmann G, Huang J, Wu S, Zhang N, Comerford SA, et al. Elucidation of a universal size-control mechanism in Drosophila and mammals. Cell. 2007;130:1120-33.

15. Zhao B, Wei X, Li W, Udan RS, Yang Q, Kim J, et al. Inactivation of YAP oncoprotein by the Hippo pathway is involved in cell contact inhibition and tissue growth control. Genes Dev. 2007;21:2747-61.

16. Lei QY, Zhang H, Zhao B, Zha ZY, Bai F, Pei XH, et al. TAZ promotes cell proliferation and epithelial-mesenchymal transition and is inhibited by the hippo pathway. Mol Cell Biol. 2008:28:2426-36.

17. Liu CY, Zha ZY, Zhou X, Zhang H, Huang W, Zhao D, et al. The hippo tumor pathway promotes TAZ degradation by phosphorylating a phosphodegron and recruiting the SCFbeta-TrCP E3 ligase. J Biol Chem. 2010;285:37159-69. 
18. Zhao B, Li L, Tumaneng K, Wang CY, Guan KL. A coordinated phosphorylation by Lats and CK1 regulates YAP stability through SCF (beta-TRCP). Genes Dev. 2010;24:72-85.

19. Hong AW, Meng Z, Guan KL. The Hippo pathway in intestinal regeneration and disease. Nat Rev Gastroenterol Hepatol. 2016;13:324-37.

20. Yu FX, Meng Z, Plouffe SW, Guan KL. Hippo pathway regulation of gastrointestinal tissues. Annu Rev Physiol. 2015;77:201-27.

21. Zhan T, Rindtorff N, Boutros M. Wht signaling in cancer. Oncogene. 2017;36:1461-73.

22. Azzolin L, Panciera T, Soligo S, Enzo E, Bicciato S, Dupont S, et al. YAP/TAZ in corporation in the b-catenin destruction complex orchestrates the Wnt response. Cell. 2014;158:157-70.

23. Cai J, Maitra A, Anders RA, Taketo MM, Pan D. b-Catenin destruction complex-independent regulation of Hippo-YAP signaling by APC in intestinal tumorigenesis. Genes Dev. 2015;29:1493-506.

24. Kang W, Tong JH, Chan AW, Lee TL, Lung RW, Leung PP, et al. Yes-associated protein 1 exhibits oncogenic property in gastric cancer and its nuclear accumulation associates with poor prognosis. Clin Cancer Res. 2011;17:2130-9.

25. Jiao S, Wang H, Shi Z, Dong A, Zhang W, Song X, et al. A peptide mimicking VGLL4 function acts as a YAP antagonist therapy against gastric cancer. Cancer Cell. 2014;25:166-80.

26. Cancer Genome Atlas Research Network. Comprehensive molecular characterization of gastric adenocarcinoma. Nature. 2014;513:202-9.

27. Di Lauro L, Vici P, Belli F, Tomao S, Fattoruso SI, Arena MG, et al. Docetaxel, oxaliplatin, and capecitabine combination chemotherapy for metastatic gastric cancer. Gastric Cancer. 2014;17:718-24.

28. Di Lauro L, Giacinti L, Arena MG, Sergi D, Fattoruso SI, Giannarelli D, et al. Phase II study of epirubicin, oxaliplatin and docetaxel combination in metastatic gastric or gastroesophageal junction adenocarcinoma. J Exp Clin Cancer Res. 2009;28:34

29. Di Lauro L, Nunziata C, Arena MG, Foggi P, Sperduti I, Lopez M. Irinotecan, docetaxel and oxaliplatin combination in metastatic gastric or gastroesophageal junction adenocarcinoma. Br J Cancer. 2007;97:593-7.

30. Di Lauro L, Belli F, Arena MG, Carpano S, Paoletti G, Giannarelli D, et al. Epirubicin, cisplatin and docetaxel combination therapy for metastatic gastric cancer. Ann Oncol. 2005;16:1498-502.

31. McShane L, Altman DG, Sauerbrei W, Taube SE, Gion M, Clark GM, Statistics Subcommittee of the NCI-EORTC Working Group on Cancer Diagnostics. Reporting recommendations for tumor MARKer prognostic studies (REMARK). Eur J Cancer. 2005;41:1690-6.

32. Buglioni S, Vici P, Sergi D, Pizzuti L, Di Lauro L, Antoniani B, et al. Analysis of the hippo transducers TAZ and YAP in cervical cancer and its microenvironment. Oncoimmunology. 2016;5:e1160187.

33. Vici P, Ercolani C, Di Benedetto A, Pizzuti L, Di Lauro L, Sperati F, et al. Topographic expression of the Hippo transducers TAZ and YAP in triplenegative breast cancer treated with neoadjuvant chemotherapy. J Exp Clin Cancer Res. 2016;35:62.

34. Di Benedetto A, Mottolese M, Sperati F, Ercolani C, Di Lauro L, Pizzuti $L$, et al. The Hippo transducers TAZ/YAP and their target CTGF in male breast cancer. Oncotarget. 2016;7:43188-98.

35. Ronchetti L, Melucci E, De Nicola F, Goeman F, Casini B, Sperati F, et al. DNA damage repair and survival outcomes in advanced gastric cancer patients treated with first-line chemotherapy. Int J Cancer. 2017;140:2587-95.

36. Song M, Cheong JH, Kim H, Noh SH, Kim H. Nuclear expression of yesassociated protein 1 correlates with poor prognosis in intestinal type gastric cancer. Anticancer Res. 2012;32:3827-34.

37. Sun L, Chen F, Shi W, Qi L, Zhao Z, Zhang J. Prognostic impact of TAZ and $\beta$-catenin expression in adenocarcinoma of the esophagogastric junction. Diagn Pathol. 2014;9:125.

38. Yue G, Sun X, Gimenez-Capitan A, Shen J, Yu L, Teixido C, et al. TAZ is highly expressed in gastric signet ring cell carcinoma. Biomed Res Int. 2014;2014:393064.

39. Miller E, Yang J, DeRan M, Wu C, Su Al, Bonamy GM, et al. Identification of serum-derived sphingosine-1-phosphate as a small molecule regulator of YAP. Chem Biol. 2012;19:955-62.
40. Mo JS, Yu FX, Gong R, et al. Regulation of the Hippo-YAP pathway by protease-activated receptors (PARs). Genes Dev. 2012;26:2138-43.

41. Wennmann DO, Vollenbroker B, Eckart AK, Bonse J, Erdmann F, Wolters DA, et al. The Hippo pathway is controlled by angiotensin II signaling and its reactivation induces apoptosis in podocytes. Cell Death Dis. 2014;5:e1519.

42. Yu FX, Zhao B, Panupinthu N, Jewell JL, Lian I, Wang LH, et al. Regulation of the Hippo-YAP pathway by G-protein-coupled receptor signaling. Cell. 2012;150:780-91.

43. Zhou X, Wang S, Wang Z, Feng X, Liu P, Lv XB, et al. Estrogen regulates Hippo signaling via GPER in breast cancer. J Clin Invest. 2015;125:2123-35.

44. Dupont S, Morsut L, Aragona M, Enzo E, Giulitti S, Cordenonsi M, et al. Role of YAP/TAZ in mechanotransduction. Nature. 2011;474:179-83.

45. Aragona M, Panciera T, Manfrin A, Giulitti S, Michielin F, Elvassore N, et al. A mechanical checkpoint controls multicellular growth through YAP/TAZ regulation by actin-processing factors. Cell. 2013;154:1047-59.

46. Wada K, Itoga K, Okano T, Yonemura S, Sasaki H. Hippo pathway regulation by cell morphology and stress fibers. Development. 2011;138:3907-14.

47. Kim NG, Koh E, Chen X, Gumbiner BM. E-cadherin mediates contact inhibition of proliferation through Hippo signaling-pathway components. Proc Natl Acad Sci USA. 2011;108:11930-5.

48. Schlegelmilch K, Mohseni M, Kirak O, Pruszak J, Rodriguez JR, Zhou D, et al. Yap1 acts downstream of a-catenin to control epidermal proliferation. Cell. 2011;144:782-95.

49. Silvis MR, Kreger BT, Lien WH, Klezovitch O, Rudakova GM, Camargo FD, et al. a-Catenin is a tumor suppressor that controls cell accumulation by regulating the localization and activity of the transcriptional coactivator Yap1. Sci Signal. 2011;4:ra33

50. Günzel D, Yu AS. Claudins and the modulation of tight junction permeability. Physiol Rev. 2013;93:525-69.

51. Chan SW, Lim CJ, Chong YF, Pobbati AV, Huang C, Hong W. Hippo pathway-independent restriction of TAZ and YAP by angiomotin. J Biol Chem. 2011;286:7018-26.

52. Wang W, Huang J, Chen J. Angiomotin-like proteins associate with and negatively regulate YAP1. J Biol Chem. 2011;286:4364-70.

53. Zhao B, Li L, Lu Q, Wang LH, Liu CY, Lei Q, et al. Angiomotin is a novel Hippo pathway component that inhibits YAP oncoprotein. Genes Dev. 2011:25:51-63.

54. Adler JJ, Johnson DE, Heller BL, Bringman LR, Ranahan WP, Conwell MD, et al. Serum deprivation inhibits the transcriptional co-activator YAP and cell growth via phosphorylation of the 130-kDa isoform of angiomotin by the LATS1/2 protein kinases. Proc Natl Acad Sci USA. 2013;110:17368-73.

55. Hirate Y, Hirahara S, Inoue K, Suzuki A, Alarcon VB, Akimoto K, et al. Polarity-dependent distribution of angiomotin localizes Hippo signaling in preimplantation embryos. Curr Biol. 2013;23:1181-94.

56. Wang W, Huang J, Wang X, Yuan J, Li X, Feng L, et al. PTPN14 is required for the density-dependent control of YAP1. Genes Dev. 2012;26:1959-71.

57. Liu X, Yang N, Figel SA, Wilson KE, Morrison CD, Gelman IH, et al. PTPN14 interacts with and negatively regulates the oncogenic function of YAP. Oncogene. 2013;32:1266-73.

58. Zhang T, Zhang J, You X, Liu Q, Du Y, Gao Y, et al. Hepatitis B virus X protein modulates oncogene yes-associated protein by CREB to promote growth of hepatoma cells. Hepatology. 2012;56:2051-9.

59. Liu P, Zhang H, Liang $X$, Ma H, Luan F, Wang B, et al. HBV preS2 promotes the expression of TAZ via miRNA-338-3p to enhance the tumorigenesis of hepatocellular carcinoma. Oncotarget. 2015;6:29048-59.

60. He C, Mao D, Hua G, Lv X, Chen X, Angeletti PC, et al. The Hippo/YAP pathway interacts with EGFR signaling and HPV oncoproteins to regulate cervical cancer progression. EMBO Mol Med. 2015;7:1426-49.

61. Yu FX, Zhao B, Guan KL. Hippo pathway in organ size control, tissue homeostasis, and cancer. Cell. 2015;163:811-28.

62. MacDonald BT, Tamai K, He X. Wnt/beta-catenin signaling: components, mechanisms, and diseases. Dev Cell. 2009;17:9-26. 